Internat. J. Math. \& Math. Sci.

Vol. 24, No. 12 (2000) 857-863

S0161171200004282

(C) Hindawi Publishing Corp.

\title{
ABOUT THE EXISTENCE OF THE THERMODYNAMIC LIMIT FOR SOME DETERMINISTIC SEQUENCES OF THE UNIT CIRCLE
}

\author{
STEFANO SIBONI
}

(Received 20 December 1999)

\begin{abstract}
We show that in the set $\Omega=\mathbb{R}_{+} \times(1,+\infty) \subset \mathbb{R}_{+}^{2}$, endowed with the usual

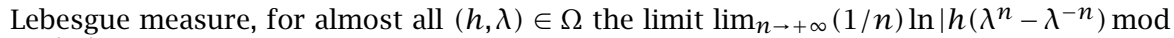
$\left[-\frac{1}{2}, \frac{1}{2}\right) \mid$ exists and is equal to zero. The result is related to a characterization of relaxation to equilibrium in mixing automorphisms of the two-torus. It is nothing but a curiosity, but maybe you will find it nice.
\end{abstract}

Keywords and phrases. Thermodynamic limit, Borel-Cantelli argument.

2000 Mathematics Subject Classification. Primary 11B83; Secondary 37A25.

1. Introduction. In the analysis of relaxation to equilibrium of mixing automorphisms of the two-torus [1, 2, 3] one encounters the following problem. Suppose that the one-torus is parameterized by the unit interval $\left[-\frac{1}{2}, \frac{1}{2}\right)$ and for appropriate constants $h>0$ and $\lambda>1$ consider the real sequence

$$
x_{n}=h\left(\lambda^{n}-\lambda^{-n}\right) \bmod \left[-\frac{1}{2}, \frac{1}{2}\right) \quad \forall n \in \mathbb{N} .
$$

A significant definition of an exponential "relaxation rate" can be given if the so-called "thermodynamic" limit [3],

$$
\lim _{n \rightarrow+\infty}-\frac{1}{n} \ln \left|x_{n}\right|
$$

exists and is equal to zero. Existence of (1.2) is clearly not obvious, since the $x_{n}$ 's typically wander through the whole interval $\left[-\frac{1}{2}, \frac{1}{2}\right)$ but every so often they visit a small neighborhood of zero, where the logarithm is singular. Actually, not even if one replaces the ordinary limit in (1.2) with a supremum limit the finiteness of the result is assured.

This note is devoted to a measure theoretical discussion of the previous problem. One can show that existence to zero of limit (1.2) occurs almost surely, for almost any choice of the parameters $h$ and $\lambda$, with respect to a measure suitably defined.

2. Results. Our goal is to prove the statement below.

THEOREM 2.1. Consider the set $\Omega=\mathbb{R}_{+} \times(1,+\infty) \subset \mathbb{R}_{+}^{2}$ endowed with the usual Lebesgue measure $\mu$. Then, for $\mu$ almost all $(h, \lambda) \in \Omega$ there holds

$$
\lim _{n \rightarrow+\infty} \frac{1}{n} \ln \left|h\left(\lambda^{n}-\lambda^{-n}\right) \bmod \left[-\frac{1}{2}, \frac{1}{2}\right)\right|=0 .
$$


This result can be easily deduced by means of standard arguments of measure theory once the following main theorem is proved.

THEOREM 2.2. Let $h>0$ and $Q \in \mathbb{N}, Q>1$, some fixed constants. Consider the set $G$ of all $\lambda \in[1, Q]$ for which a (possibly $\lambda$-dependent) real sequence $\left(a_{n}\right)_{n \in \mathbb{N}}$ and an integer $n^{\prime} \in \mathbb{N}$ exist such that

(a) $a_{n}>0 \forall n>n^{\prime}$;

(b) $a_{n} \leq\left|h\left(\lambda^{n}-\lambda^{-n}\right) \bmod \left[-\frac{1}{2}, \frac{1}{2}\right)\right| \forall n>n^{\prime}$;

(c) $\lim _{n \rightarrow+\infty}(1 / n) \ln a_{n}=0$.

Then, if $\mu$ denotes the Lebesgue measure on $\mathbb{R}$ :

(1) the set $G \subseteq[1, Q]$ is actually nonempty;

(2) $G$ is $\mu$-measurable and its measure holds $\mu(G)=Q-1$.

As a consequence, the set $B=[1, Q] \backslash G$, where conditions (a), (b), and (c) are not simultaneously satisfied, is also $\mu$-measurable and of vanishing measure.

We firstly prove the result by considering values of $\lambda$ in the interval $[1+\eta, Q]$, with $\eta$ small positive number arbitrarily fixed $(\eta<1 / 2)$. We therefore look for the subset $G_{\eta}$ of $\lambda \in[1+\eta, Q]$, where hypotheses (a), (b), and (c) are satisfied by a suitable choice of the sequence $\left(a_{n}\right)_{n \in \mathbb{N}}$ and of the integer $n^{\prime} \in \mathbb{N}$. The basic idea of the proof is that the $\mu$-measure of $G_{\eta}$ turns out to be $Q-1-\eta$ even if we confine ourselves to choose the sequence $\left(a_{n}\right)_{n \in \mathbb{N}}$ in the form

$$
a_{n}=\frac{1}{n^{2}} \quad \forall n \in \mathbb{N},
$$

which certainly fulfills requirements (a) and (c), and enable us to deal with the only condition (b) on $\lambda$.

Let us then take $a_{n}=1 / n^{2}$ for all $n \in \mathbb{N}$ and an arbitrarily given value of $n \in \mathbb{N}$. Before tackling the real proof, we need some definitions.

DEFINITION 2.3. We introduce the set $B_{n} \subseteq[1+\eta, Q]$

$$
B_{n}=\left\{\lambda \in[1+\eta, Q]: a_{n}>\left|h\left(\lambda^{n}-\lambda^{-n}\right) \bmod \left[-\frac{1}{2}, \frac{1}{2}\right)\right|\right\},
$$

that is, the set of $\lambda \in[1+\eta, Q]$, where the condition $a_{n} \leq\left|h\left(\lambda^{n}-\lambda^{-n}\right) \bmod \left[-\frac{1}{2}, \frac{1}{2}\right)\right|$ is not satisfied for the assigned $n \in \mathbb{N}$.

Notice that $B_{n}$ is a finite union of intervals because the function $\Phi_{n}(\lambda)=\lambda^{n}-\lambda^{-n}$ is strictly increasing in $[+1,+\infty)$ at fixed $n$. In fact

$$
\Phi_{n}^{\prime}(\lambda)=n\left(\lambda^{n-1}+\lambda^{-(n+1)}\right)>0 \quad \forall \lambda \in[1,+\infty) .
$$

Consequently, $B_{n}$ is $\mu$-measurable as a finite union of bounded intervals.

DEFINITION 2.4. We further introduce the set $\hat{B}_{n^{\prime}} \subseteq[1+\eta, Q], n^{\prime} \in \mathbb{N}$, given by

$$
\hat{B}_{n^{\prime}}=\left\{\lambda \in[1+\eta, Q]: a_{n}>\left|h\left(\lambda^{n}-\lambda^{-n}\right) \bmod \left[-\frac{1}{2}, \frac{1}{2}\right)\right|, n>n^{\prime}\right\}=\bigcup_{n>n^{\prime}} B_{n}
$$

which is obviously $\mu$-measurable as a countable union of $\mu$-measurable sets. 
DEFINITION 2.5. We finally introduce the "bad" set $B_{\eta} \subseteq[1+\eta, Q]$

$$
B_{\eta}=\bigcap_{n^{\prime}=1}^{\infty} \hat{B}_{n^{\prime}},
$$

where condition (b) is not satisfied-with this particular choice of the sequence $\left(a_{n}\right)_{n \in \mathbb{N}} . B_{\eta}$ is also a $\mu$-measurable set, as a countable intersection of $\mu$-measurable sets.

An immediate consequence of the previous definitions is that $[1+\eta, Q] \backslash G_{\eta}=B_{\eta}$. Our goal is to prove that $\mu\left(B_{\eta}\right)=0$. To this end, since for all $n^{\prime} \in \mathbb{N}, B_{\eta} \subseteq \hat{B}_{n^{\prime}}$ by definition, it is enough to show that

$$
\lim _{n^{\prime} \rightarrow+\infty} \mu\left(\hat{B}_{n^{\prime}}\right)=0 .
$$

Therefore, we can confine ourselves to consider values of $n^{\prime} \in \mathbb{N}$ large enough, and owing to Definition 2.4, we can also assume values of $n \in \mathbb{N}$ greater that $n^{\prime}$. More precisely, we impose the following technical requirements on the size of $n^{\prime}$ and $n$. We take $n>n^{\prime} \in \mathbb{N}$ such that:

(i) $a_{n^{\prime}}=1 / n^{\prime 2}<\eta \Rightarrow a_{n}<\eta \forall n>n^{\prime}$.

(ii) $a_{n}-h /(1+\eta)^{n}=1 / n^{2}-h /(1+\eta)^{n}>0 \forall n>n^{\prime}$.

(iii) $h\left[(1+\eta)^{n}-(1+\eta)^{-n}\right]>3 / 2$ and $h\left[Q^{n}-Q^{-n}\right]>5 / 2 \forall n>n^{\prime}$.

Under the previous conditions we can state the following lemmas.

LEMMA 2.6. The $\mu$-measure of $B_{n}, n$ as above, admits the upper bound

$$
\mu\left(B_{n}\right) \leq 2 \varepsilon_{n}\left(\frac{1}{h}\right)^{1 / n}\left[h\left(Q^{n}-Q^{-n}\right)\right]^{1 / n},
$$

where $\varepsilon_{n}=a_{n}+h /(1+\eta)^{n}>0$.

Proof. We firstly notice that $1+a_{n}<1+\eta$ by (i); on the other hand, since $\eta<1 / 2$ by hypothesis, (i) implies $a_{n}<1 / 2$, so that all the intervals $\left(p-a_{n}, p+a_{n}\right), p=$ $2, \ldots,\left\lfloor h\left(Q^{n}-Q^{-n}\right)\right\rfloor+1$ are disjoint.

By using (iii) and denoted with $I_{n}$ the integer set $\left\{2,3, \ldots,\left\lfloor h\left(Q^{n}-Q^{-n}\right)\right\rfloor+1\right\}$, we deduce

$$
\begin{aligned}
B_{n} & \subseteq\left\{\lambda \in[1+\eta, Q]: h\left(\lambda^{n}-\lambda^{-n}\right) \in \bigcup_{p=2}^{\left\lfloor h\left(Q^{n}-Q^{-n}\right)\right\rfloor+1}\left(p-a_{n}, p+a_{n}\right)\right\} \\
& =\left\{\lambda \in[1+\eta, Q]: p-a_{n}<h\left(\lambda^{n}-\lambda^{-n}\right)<p+a_{n}, p \in I_{n}\right\} \\
& =\left\{\lambda \in[1+\eta, Q]: p-\left(a_{n}-h \lambda^{-n}\right)<h \lambda^{n}<p+a_{n}+h \lambda^{-n}, p \in I_{n}\right\} .
\end{aligned}
$$

Now it is clear that for all $\lambda \in[1+\eta, Q]$,

$$
a_{n}-\frac{h}{(1+\eta)^{n}} \leq a_{n}-h \lambda^{-n}<a_{n}+h \lambda^{-n} \leq a_{n}+\frac{h}{(1+\eta)^{n}}
$$

and by (ii),

$$
a_{n}-\frac{h}{(1+\eta)^{n}}>0
$$


from which we obtain

$$
0<a_{n}-\frac{h}{(1+\eta)^{n}} \leq a_{n}-h \lambda^{-n}<a_{n}+h \lambda^{-n} \leq a_{n}+\frac{h}{(1+\eta)^{n}} \quad \forall \lambda \in[1+\eta, Q] .
$$

By enlarging each covering interval in (2.9), we are then led to the inclusion

$$
B_{n} \subseteq\left\{\lambda \in[1+\eta, Q]: p-\left(a_{n}+\frac{h}{(1+\eta)^{n}}\right)<h \lambda^{n}<p+a_{n}+\frac{h}{(1+\eta)^{n}}, p \in I_{n}\right\}
$$

and recalling the definition of $\varepsilon_{n}$,

$$
\begin{aligned}
B_{n} & \subseteq\left\{\lambda \in[1+\eta, Q]:\left(\frac{p-\varepsilon_{n}}{h}\right)^{1 / n}<\lambda<\left(\frac{p+\varepsilon_{n}}{h}\right)^{1 / n}, p \in I_{n}\right\} \\
& \subseteq \bigcup_{p=2}^{\left\lfloor h\left(Q^{n}-Q^{-n}\right)\right\rfloor+1}\left(\left(\frac{p-\varepsilon_{n}}{h}\right)^{1 / n},\left(\frac{p+\varepsilon_{n}}{h}\right)^{1 / n}\right),
\end{aligned}
$$

the final set being $\mu$-measurable as a finite union of intervals. Whence

$$
\mu\left(B_{n}\right) \leq \sum_{p=2}^{\left\lfloor h\left(Q^{n}-Q^{-n}\right)\right\rfloor+1}\left(\frac{1}{h}\right)^{1 / n}\left[\left(p+\varepsilon_{n}\right)^{1 / n}-\left(p-\varepsilon_{n}\right)^{1 / n}\right] .
$$

Moreover, for all $p=2,3, \ldots,\left\lfloor h\left(Q^{n}-Q^{-n}\right)\right\rfloor+1$ Lagrange mean value theorem implies the equalities below

$$
\left(p+\varepsilon_{n}\right)^{1 / n}-\left(p-\varepsilon_{n}\right)^{1 / n}=\frac{1}{n}\left(p+\xi_{p}\right)^{(1 / n)-1} 2 \varepsilon_{n}
$$

for some $\xi_{p} \in\left(-\varepsilon_{n}, \varepsilon_{n}\right)$, and since

$$
\left(p+\xi_{p}\right)^{(1 / n)-1}=\frac{1}{\left(p+\xi_{p}\right)^{1-(1 / n)}} \leq \frac{1}{(p-1)^{1-(1 / n)}}
$$

we conclude that

$$
\begin{aligned}
\mu\left(B_{n}\right) & \leq \sum_{p=2}^{\left\lfloor h\left(Q^{n}-Q^{-n}\right)\right\rfloor+1}\left(\frac{1}{h}\right)^{1 / n} \frac{1}{n} \frac{1}{(p-1)^{1-(1 / n)}} 2 \varepsilon_{n} \\
& =\frac{2 \varepsilon_{n}}{n}\left(\frac{1}{h}\right)^{1 / n\left\lfloor h\left(Q^{n}-Q^{-n}\right)\right\rfloor} \sum_{p=1}^{1} \frac{1}{p^{1-(1 / n)}} .
\end{aligned}
$$

As $\left(p^{1-(1 / n)}\right)^{-1}$ is a decreasing function of $p$, the following upper estimate holds

$$
\begin{aligned}
\sum_{p=1}^{\left\lfloor h\left(Q^{n}-Q^{-n}\right)\right\rfloor} \frac{1}{p^{1-(1 / n)}} & \leq \int_{0}^{\left\lfloor h\left(Q^{n}-Q^{-n}\right)\right\rfloor} p^{(1 / n)-1} d p=\left[n p^{1 / n}\right]_{0}^{\left\lfloor h\left(Q^{n}-Q^{-n}\right)\right\rfloor} \\
& =n\left\lfloor h\left(Q^{n}-Q^{-n}\right)\right\rfloor^{1 / n}
\end{aligned}
$$

and finally $\mu\left(B_{n}\right) \leq 2 \varepsilon_{n}(1 / h)^{1 / n}\left[h\left(Q^{n}-Q^{-n}\right)\right]^{1 / n}$, which completes the proof. 
LEMMA 2.7. If $n^{\prime}>0$ (satisfying (i), (ii), and (iii)) is sufficiently large, the $\mu$-measure of $\hat{B}_{n^{\prime}}$ is bounded by

$$
\mu\left(\hat{B}_{n^{\prime}}\right) \leq 2(Q+\varepsilon) \sum_{n=n^{\prime}+1}^{\infty} \varepsilon_{n}
$$

for some small $\varepsilon>0$.

Proof. Because of the identity $\hat{B}_{n^{\prime}}=\cup_{n>n^{\prime}} B_{n}$ and using Lemma 2.6, we have the following estimate

$$
\mu\left(\hat{B}_{n^{\prime}}\right) \leq \sum_{n=n^{\prime}+1}^{\infty} \mu\left(B_{n}\right) \leq 2 \sum_{n=n^{\prime}+1}^{\infty} \varepsilon_{n}\left(\frac{1}{h}\right)^{1 / n}\left[h\left(Q^{n}-Q^{-n}\right)\right]^{1 / n} .
$$

Notice that for all $h>0$, and $Q \in \mathbb{N}, Q>1$

$$
\lim _{n \rightarrow+\infty}\left(\frac{1}{h}\right)^{1 / n}\left[h\left(Q^{n}-Q^{-n}\right)\right]^{1 / n}=Q
$$

so that for some $\varepsilon>0, \varepsilon \ll Q$, and $n^{\prime}$ sufficiently large there holds

$$
Q-\varepsilon<\left(\frac{1}{h}\right)^{1 / n}\left[h\left(Q^{n}-Q^{-n}\right)\right]^{1 / n}<Q+\varepsilon \quad \forall n \in \mathbb{N}, n>n^{\prime} .
$$

Whence for $n^{\prime}$ as above

$$
\mu\left(\hat{B}_{n^{\prime}}\right) \leq 2 \sum_{n=n^{\prime}+1}^{\infty} \varepsilon_{n}(Q+\varepsilon)=2(Q+\varepsilon) \sum_{n=n^{\prime}+1}^{\infty} \varepsilon_{n}
$$

which is finite, owing to

$$
\sum_{n=1}^{\infty} \varepsilon_{n}=\sum_{n=1}^{\infty}\left(\frac{1}{n^{2}}+\frac{h}{(1+\eta)^{n}}\right)=\frac{\pi^{2}}{6}+\frac{h}{\eta} .
$$

LEMMA 2.8. The measure of $B_{\eta}$ is zero

$$
\mu\left(B_{\eta}\right)=0 .
$$

Proof. Since for all $n^{\prime} \in \mathbb{N}$ we have that $B_{\eta} \subseteq \hat{B}_{n^{\prime}}$, in particular this will be true for all $n^{\prime} \in \mathbb{N}$ large enough to satisfy the requirements of the previous lemmas. Thus

$$
\mu\left(B_{\eta}\right) \leq \mu\left(\hat{B}_{n^{\prime}}\right) \leq 2(Q+\varepsilon) \sum_{n=n^{\prime}+1}^{\infty} \varepsilon_{n}
$$

and therefore

$$
\mu\left(B_{\eta}\right) \leq \lim _{n^{\prime} \rightarrow+\infty} 2(Q+\varepsilon) \sum_{n=n^{\prime}+1}^{\infty} \varepsilon_{n},
$$

where the limit is obviously zero, because of $\sum_{n=1}^{\infty} \varepsilon_{n}<+\infty$. By the nonnegativity of measure we have the result. 
Proof OF TheOrem 2.2. As a consequence of Lemma 2.8, the "good" set $G_{\eta}=$ $[1+\eta, Q] \backslash B_{\eta}$ of $\lambda$-values in $[1+\eta, Q]$ satisfying condition (b) for the particular choice of $\left(a_{n}\right)_{n \in \mathbb{N}}, a_{n}=1 / n^{2}$, is of course $\mu$-measurable and with Lebesgue measure

$$
\mu\left(G_{\eta}\right)=Q-1-\eta-\mu\left(B_{\eta}\right)=Q-1-\eta .
$$

If we now consider an arbitrary choice of the sequence $\left(a_{n}\right)_{n \in \mathbb{N}}$, compatible again with conditions (a) and (c), the previous set $G_{\eta}$ will maybe "grow" by a subset $\tilde{G}_{\eta} \subseteq$ $[1+\eta, Q] \backslash G_{\eta}:$

$$
G_{\eta} \longrightarrow G_{\eta}^{o}=G_{\eta} \cup \tilde{G}_{\eta}
$$

But as $\mu\left([1+\eta, Q] \backslash G_{\eta}\right)=0$ it follows that $\tilde{G}_{\eta}$ is also $\mu$-measurable and of vanishing $\mu$-measure. Hence we finally conclude that the full set $G_{\eta}^{o}$, corresponding to arbitrary (a)- and (c)-conditioned sequences $\left(a_{n}\right)_{n \in \mathbb{N}}$, is $\mu$-measurable with measure

$$
\mu\left(G_{\eta}^{o}\right)=Q-1-\eta
$$

and that the corresponding full set $B_{\eta}^{o}=[1+\eta, Q] \backslash G_{\eta}^{o}$ of $\lambda$ values where condition (b) is not fulfilled for any (a)- and (c)-conditioned sequence $\left(a_{n}\right)_{n \in \mathbb{N}}$ is in turn $\mu$-measurable with vanishing $\mu$-measure:

$$
\mu\left(B_{\eta}^{o}\right)=Q-1-\eta-\mu\left(G_{\eta}^{o}\right)=0 .
$$

So far we have proved that $B_{\eta}$ is a set of vanishing measure in any closed interval $[1+\eta, Q]$ with $\eta>0$. Consider now $B, G$ in $[1, Q]$, that is, according to the previous notation

$$
B=B_{0}, \quad G=G_{0} .
$$

We firstly notice that $B$ and $G$ are both $\mu$-measurable because

$$
G=\bigcup_{n^{\prime}=1}^{\infty} \bigcap_{n>n^{\prime}} S_{n},
$$

where $S_{n}$ is the finite union of subintervals in [1,Q] (dependent on $n$ ), and $B=$ $[1, Q] \backslash G$. Then take

$$
B=(B \cap[1,1+\eta)) \cup(B \cap[1+\eta, Q])
$$

union of disjoint sets, for some fixed $\eta \in\left(0, \frac{1}{2}\right)$. The $\mu$-measurable set $B \cap[1+\eta, Q]$ is the "bad" set in $[1+\eta, Q]$, so that by Lemma 2.8 ,

$$
\mu(B \cap[1+\eta, Q])=0 .
$$

As for the $\mu$-measurable set $B \cap[1,1+\eta)$, we have the identity

$$
\begin{aligned}
B \cap[1,1+\eta) & =B \cap\left(\{1\} \cup \bigcup_{k=1}^{\infty}\left[1+\frac{\eta}{k+1}, 1+\frac{\eta}{k}\right)\right) \\
& =B \cap\{1\} \cup \bigcup_{k=1}^{\infty} B \cap\left[1+\frac{\eta}{k+1}, 1+\frac{\eta}{k}\right)
\end{aligned}
$$


union of disjoint sets. But the $\mu$-measurable set

$$
B \cap\left[1+\frac{\eta}{k+1}, 1+\frac{\eta}{k}\right), \quad k \in \mathbb{N} \backslash\{0\},
$$

satisfies

$$
B \cap\left[1+\frac{\eta}{k+1}, 1+\frac{\eta}{k}\right) \subseteq B \cap\left[1+\frac{\eta}{k+1}, Q\right]
$$

and since $1 / 2>\eta /(k+1)>0$ for any given $k \in \mathbb{N} \backslash\{0\}$, we obtain

$$
\mu\left(B \cap\left[1+\frac{\eta}{k+1}, 1+\frac{\eta}{k}\right)\right)=0 .
$$

On the other hand, there trivially holds $\mu(B \cap\{1\})=0$, so that

$$
\mu(B \cap[1,1+\eta))=\mu(B \cap\{1\})+\sum_{k=1}^{\infty} \mu\left(B \cap\left[1+\frac{\eta}{k+1}, 1+\frac{\eta}{k}\right)\right)=0 .
$$

Whence, finally,

$$
\mu(B)=\mu(B \cap[1,1+\eta))+\mu(B \cap[1+\eta, Q))=0,
$$

that is, $\mu(B)=0$ and $\mu(G)=Q-1$. The proof is complete.

\section{REFERENCES}

[1] A. Bazzani, S. Siboni, G. Turchetti, and S. Vaienti, From dynamical systems to local diffusion processes, Chaotic Dynamics (Patras, 1991), pp. 139-144, NATO Adv. Sci. Inst. Ser. B Phys., vol. 298, Plenum, New York, 1992. CMP 1209873.

[2] S. Siboni, Rilassamento all'equilibrio in un sistema mixing e analisi di un modello di diffusione modulata, Tesi di Dottorato di Ricerca dell'Università degli Studi, Bologna (Italy), 1991.

[3] S. Siboni, G. Turchetti, and S. Vaienti, Thermodynamic limit and relaxation to equilibrium in toral area-preserving transformations, Jour. Stat. Phys. 75 (1994), no. 1-2, 167.

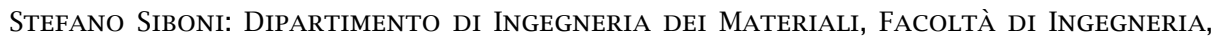
UNIVERSITÀ DI TRENTO, MESIANO 38050, TRENTO, ITALY

E-mail address: stefano.siboni@ing.unitn.it 


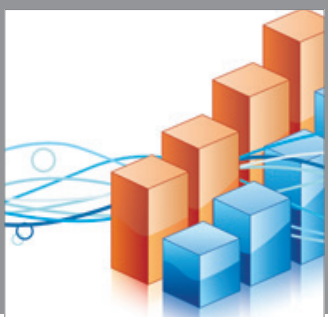

Advances in

Operations Research

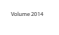



\section{The Scientific} World Journal
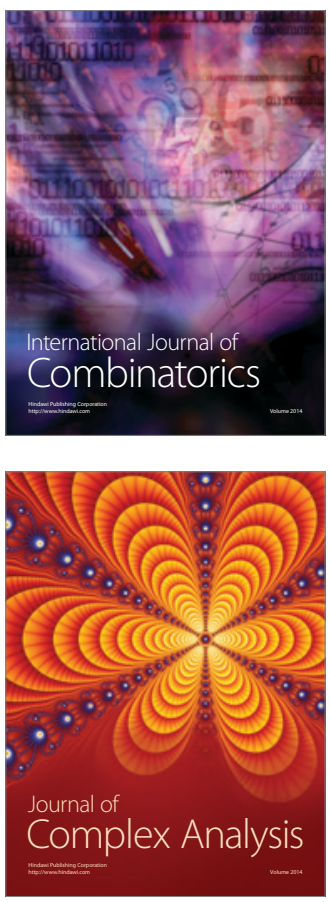

International Journal of

Mathematics and

Mathematical

Sciences
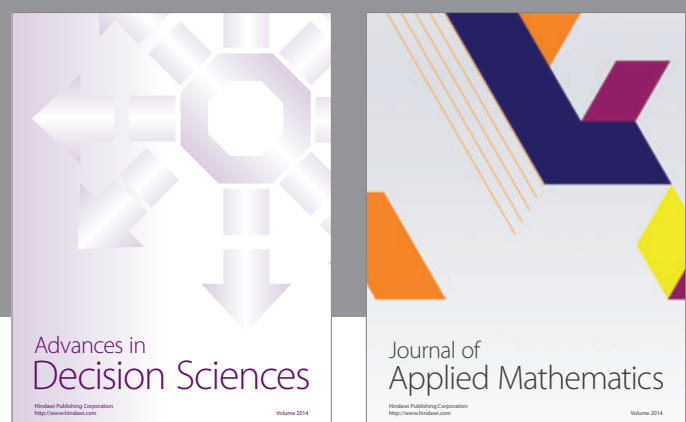

Journal of

Applied Mathematics
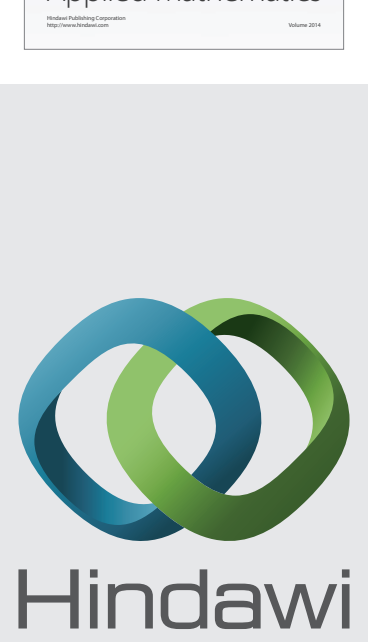

Submit your manuscripts at http://www.hindawi.com
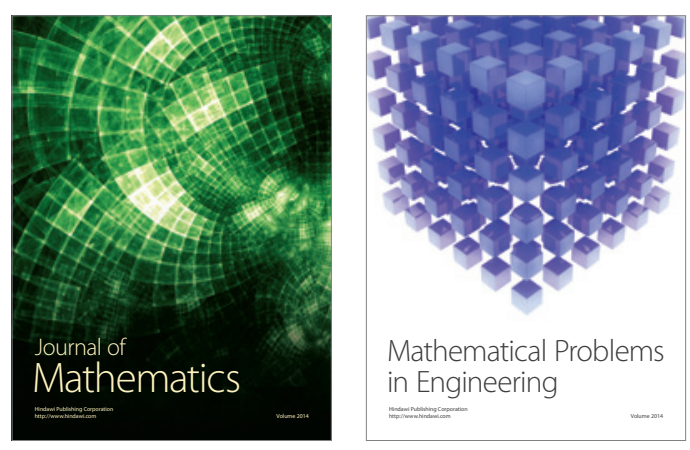

Mathematical Problems in Engineering
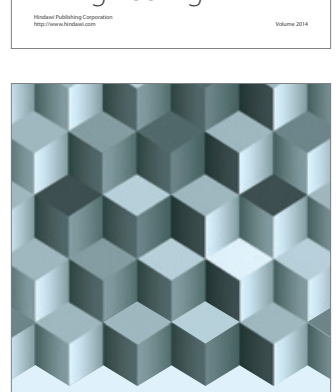

Journal of

Function Spaces
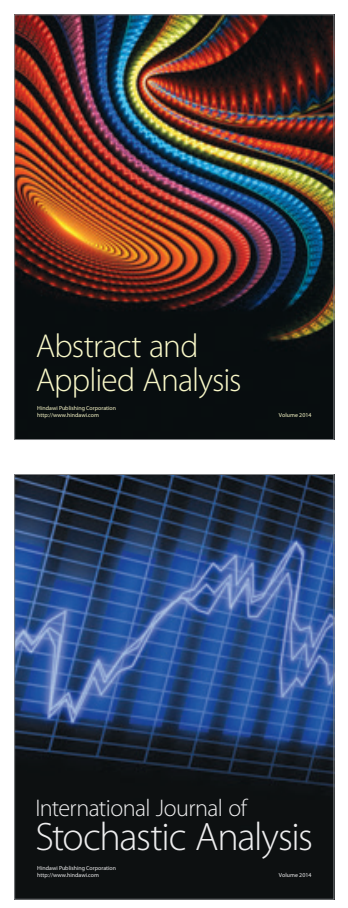

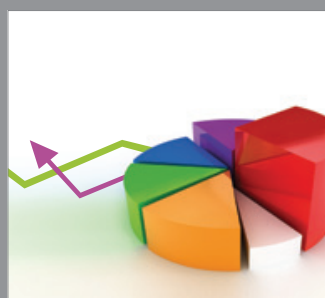

ournal of

Probability and Statistics

Promensencen
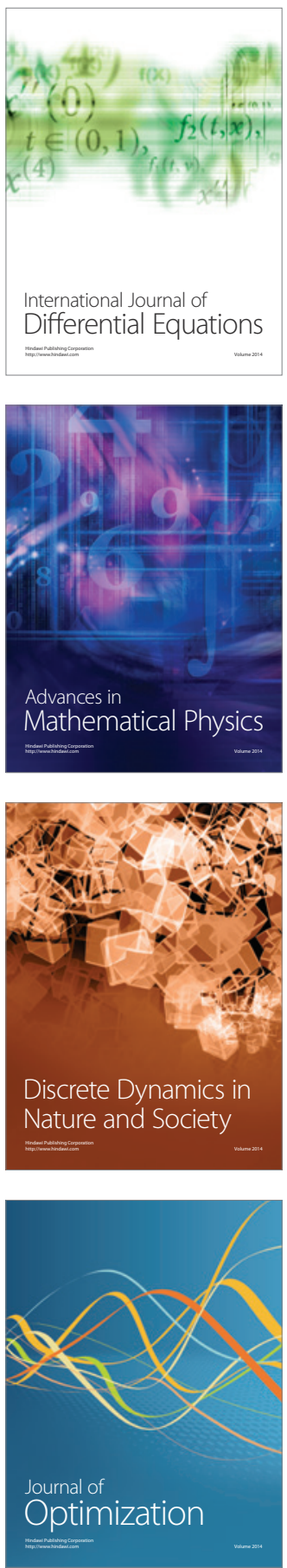